\title{
REPRESENTACIÓN SOCIAL DE LOS ESTUDIANTES DE MAGISTERIO SOBRE LOS MAYORES: UN ESTUDIO PILOTO
}

\author{
Antonio Matas Terrón ${ }^{1}$ \\ Juan José Leiva Olivencia \\ Pablo D. Franco Caballero \\ Estela Isequilla Alarcón \\ Universidad de Málaga, Málaga, España
}

\begin{abstract}
Resumen. El estudio trató de conocer la imagen subjetiva que tienen los estudiantes de magisterio de la Universidad de Málaga sobre los adultos mayores. El objetivo pretende identificar sesgos en las teorías implícitas de los estudiantes para trata de poner en marcha programas formativos que los corrijan. Todo ello está basado en la idea de que la situación de los adultos mayores se debe, en gran medida, al contexto cultural. Se llevó a cabo un estudio exploratorio bajo un diseño de encuesta, usando una escala tipo Likert. En el estudio participó una muestra incidental de 169 estudiantes. Se realizó un análisis descriptivo por ítem, así como diversos contrastes de hipótesis en función del sexo y de la experiencia en el cuidado y convivencia con adultos mayores. Los resultados muestran un predominio de la percepción negativa, principalmente en las dimensiones de relaciones sociales y salud. Se reflexiona sobre cómo esta percepción podría afectar al bienestar de los adultos mayores actuales y en el futuro, así como de la necesidad de que los educadores reciban una formación especializada para atender la realidad de este segmento de población. En este sentido, se plantea la necesidad de incrementar la formación pedagógica de los profesionales de la educación pata atender de forma adecuada las necesidades del aprendizaje a lo largo de la vida.
\end{abstract}

Palabras clave: representación social, estudiantes universitarios, vejez, actitudes, adultos mayores.

\section{SOCIAL REPRESENTATION OF ELDERLY IN STUDENT TEACHERS: A PILOT STUDY}

\begin{abstract}
This study analyzes the subjective image that the students of education from the University of Malaga have about older adults. The study aims to identify biases in students' implicit theories to launch training programs that corrected them. The assumption here is that the situation of older adults is due, largely, to the cultural context. We carried out an exploratory study and a survey using a Likert scale. An incidental sample of 169 students participated in the study. We performed a descriptive analysis by item, as well as various hypothesis contrasting on gender and experience in coexistence and caring older adults. The results show a predominance of a negative perception, mainly in the dimensions of social relationships and health. The article reflects on how this perception could affect the well-being of current and future generations of older people and the need that educators receive specialized training to meet their reality. In this sense, it is needed more pedagogical training for the educators in order to meet the needs of their lifelong learning.
\end{abstract}

Keywords: social representation, university students, age, attitudes, elderly.

\footnotetext{
${ }^{1}$ Antonio Matas Terrón. Departamento de Teoría e Historia de la Educación y Métodos de Investigación y Diagnóstico en Educación. Universidad de Málaga. Facultad de Ciencias de la Educación. Desp. 6.08. Campus de Teatinos, C.P. 29071-Málaga. amatas@uma.es
} 


\title{
REPRESENTAÇÃO SOCIAL DOS ALUNOS DE MAGISTÉRIO SOBRE OS IDOSOS: UM ESTUDO PILOTO
}

\begin{abstract}
Resumo. Este estudo tratou de conhecer a imagem subjetiva de estudantes de magistério da Universidade de Málaga sobre as pessoas de terceira idade. O objetivo visa identificar preconceitos implícitos para desenvolver programas com o propósito de promover mudanças. Tudo isso é baseado na ideia de que a situação dos idosos é resultado, em grande parte, do contexto cultural. Foi realizado um estudo exploratório, utilizando-se uma escala tipo Likert. Do estudo participou uma amostra incidental de 169 alunos. Foi realizada uma análise descritiva por item, bem como vários contrastes de hipótese em função do sexo e da experiência no cuidado e convivência com pessoas idosas. Os resultados mostram uma predominância da percepção negativa, principalmente nas dimensões relações sociais e saúde, indicando a necessidade de refletir sobre como essa percepção pode afetar o bem-estar dos idosos no presente e no futuro, bem como a de oferecer aos educadores uma formação especializada para atender a realidade desse segmento da população. Nesse sentido, observa-se a relevância de incrementar a formação pedagógica dos profissionais da educação para atender de forma adequada as necessidades de aprendizagem ao longo da vida.
\end{abstract}

Palavras-chave: representação social, estudantes universitários, velhice, atitudes, pessoas de terceira idade.

\section{Introducción}

Según todas las estadísticas y simulaciones a corto y medio plazo, la población española está sufriendo un sobre-envejecimiento (Abades \& Rayón, 2012). Según estas previsiones en los próximos quince años, en España residirían once millones trescientas mil personas mayores de sesenta y cuatro años (un treinta y cuatro por ciento más que ahora). Esta cifra se incrementaría hasta casi los dieciséis millones de personas en cincuenta años. Mientras, los índices de natalidad sugerían cayendo, en orden de casi un veinticinco por ciento menos en los próximos quince años (INE, 2014).

El aumento de la esperanza de vida también se está viendo acompañado de una mejor salud física, junto con mayores índices de calidad de vida (Abellán, Vilches, \& Pujol, 2014) lo que tiene implicaciones tanto para la persona, como para el entramado social, cultural y económico (Futurage, 2011). Esta nueva realidad actual determinará las demandas sociales del futuro, y entre ellas las educativas. Sin embargo, aunque no son muchos los estudios realizados sobre cómo son vistos los mayores de 65 años (a partir de aquí denominados como "adultos mayores" siguiendo las sugerencias de las Naciones Unidas para los países desarrollados) por otros segmentos de edad de la población, los estudios realizados llegan a la conclusión de que las imágenes de los adultos mayores en el resto de ciudadanos es bastante distinta a la propia imagen que los adultos mayores tiene sobre sí mismos (Adelantado, Segura, De Andrés, Feliú \& 
Martínez, 2004; Tan, Zhang \& Fan, 2004; Arnold-Cathalifaud, Thumala, Urquiza \& Ojeda, 2007) si bien, algunos estudios exploratorios han encontrado una percepción no tan negativa sobre los mayores (Aristizábal-Vallejo, 2005). Esto podría explicarse por las políticas en los países desarrollados para potenciar una sociedad del bienestar (Lachman \& Yun, 2006; Zhou, 2007). Sin embargo, parece que predomina la visión negativa entre los jóvenes, aunque estaría mediada por la formación universitaria. Así, personas con una formación vinculada la salud y la geriatría tendrían una imagen más positiva (Birkenmaier, Damron-Rodríguez \& Rowan, 2009).

Esta imagen negativa se caracterizaría por percibir al adulto mayor como triste, inflexible, enfermo, conservadora ideológicamente, improductivo, dependiente, y con deterioro físico y mental (Tan, Zhang \& Fan, 2004; Arnold-Cathalifaud, Thumala, Urquiza \& Ojeda, 2007; Fealy, Lyons, McNamara, \& Treacy, 2012).

La imagen social, estos estereotipos, pueden tener dos efectos principales. Por un lado, que predispone a los ciudadanos en la forma de relacionarse entre ellos (Pérez Grande, García del Dujo \& Martín García, 1999). Por otro lado, estas imágenes estereotipadas pueden fomentar las profecías autocumplidad, de forma que las mismas personas mayores asuman como real e inevitable, las consecuencias de dicho estereotipo (Cherry \& Palmore, 2008; Lentini, Ruiz \& Sciopini, 2008) lo que provoca una baja autoestima en el mayor (Gómez Carroza, 2003).

Dentro de este contexto, la población de estudiantes de Educación es especialmente sensible porque en el futuro inmediato serán agentes activos en el sistema educativo. A esto hay que unir que de forma inmediata es necesario formar a educadores que satisfagan las nuevas necesidades formativas de este segmento de población (Futurage, 2011; Matas, Leiva \& Franco, 2016). Dadas las consecuencias de un estereotipo sesgado y negativo sobre el adulto mayor, sería necesario desarrollar programas formativos específicos para los futuros educadores que garanticen un profesional objetivo con la realidad de esta población (Díaz, Fernández, Gázquez, González, Pérez-Fuentes, \& Ruíz, 2009; Van Dussen \& Weaver, 2009).

El objetivo de esta investigación es tratar de indagar sobre el perfil subjetivo que el alumnado universitario de Ciencias de la Educación tiene sobre el adulto mayor. con relación a los aspectos sociales, como agentes productivos, de salud y cognición. Esta imagen subjetiva o estereotipo podrá orientar sobre la puesta en marcha de programas formativos para crear especialistas en formación de adultos mayores (Gerontagogía y Gerontología educativa). A partir de los resultados, se debate sobre el tipo de formación que debería requerirse. Por otro lado, el conocer la imagen de los adultos mayores en los encuestados, permitiría identificar posibles teorías implícitas erróneas. Al mismo tiempo, esto podría usarse como punto de partida para programas educativos que corrigiesen esos errores conceptuales. 


\section{Método}

\section{Diseño y muestra}

A continuación, se presenta un estudio piloto basado en un diseño exploratorio que ha seguido una metodología de encuesta. Se ha contado con la participación de 169 estudiantes de Ciencias de la Educación de la Universidad de Málaga, a través de un muestreo incidental. La población de referencia está compuesta por 585 estudiantes con un porcentaje de mujeres estimado del $85 \%$ aproximadamente.

En la muestra obtenida, la edad media es de 21,05 años, con un mínimo de 17 y un máximo de 35 años. El porcentaje de mujeres es de $87 \%$ frente a un $13 \%$ de hombres. Todos los participantes son de la Universidad de Málaga (España).

La información se recopiló en horas de clase por parte del docente de dichas clases, administrando un cuestionario ad-hoc de 16 ítems tipo Likert, más cinco cuestiones sociodemográficas. Todos los datos se recogieron la primera semana del inicio del curso académico de 2015-16.

\section{Cuestionario y variables}

El cuestionario se ha construido a partir del diferencial semántico utilizado por Arnold-Cathalifua, Thumala, Urquiza \& Ojeda (2007). Este diferencial semántico inicial constaba de dieciséis pares de adjetivos, con una escala de elección de siete puntos: independiente/dependiente; productivo/improductivo; eficiente/ineficiente; hábil/torpe; saludable/enfermizo; sexualmente activo/sexualmente no activo; frágil/resistente; sociable/retraído; tolerante/intolerante; confiado/desconfiado; progresista/conservador; no conflictivo/conflictivo; ciudadano activo/ciudadano pasivo; protegido/desamparado; integrado/marginado; y valorado/desvalorado.

Para construir el cuestionario, dichos pares de adjetivos se vincularon a una dimensión: independencia, productividad, eficiencia, habilidad, estado de salud, sexualidad, resistencia física, sociabilidad, tolerancia, confianza, progresismo, conflictividad, actividad, protección, integración, y valoración. Para cada una de estas dimensiones se elaboraron distintos ítems tanto en sentido positivo (a favor de la dimensión) como negativo (en contra de la característica de la dimensión). De esta forma, se pretendía tener un conjunto de ítems expresados tanto a favor como en contra de la característica medida (Likert, 1932). Posteriormente, el conjunto de ítems se envió a tres jueces expertos para que valorasen su calidad (uso de un lenguaje ajustado a la población de interés, redacción clara de los ítems, y validez aparente de los ítems). Igualmente, se propusieron cuatro categorías (relaciones sociales, actividad productiva, salud y cognición) y se pidió a los jueces que asignasen cada ítem a una de esas categorías. Se eliminaron todos los ítems que presentaron problemas de clasificación o no eran aceptados por todos los jueces. Al finalizar este proceso de validación por expertos se obtuvo un cuestionario de 16 ítems tipo Likert con cinco opciones de respuesta (totalmente en desacuerdo, en desacuerdo, no estoy seguro, de acuerdo y 
totalmente de acuerdo) a los que se añadió la opción 'No tengo opinión al respecto' para evitar respuestas espurias en la opción intermedia (Baka \& Figgou, 2012).

En este proceso de construcción cabe señalar que los ítems que se asociaron finalmente a la dimensión cognitiva recogen solamente dos aspectos. Por un lado, la idea de que los mayores pueden tener una tendencia a lo innovador, al cambio, o por el contrario a lo estático, el conservadurismo; y por otro lado la idea de que las personas mayores tiendan o no a desconfiar, lo que puede estar asociado la tendencai o no al cambio/conservadurismo.

El cuestionario se completó con un con cinco preguntas sociodemográficas: edad, sexo, universidad o centro de estudios, convivencia con adultos mayores (ítem dicotómico: si /no) y experiencia en el cuidado de personas enfermas o mayores (ítem dicotómico: si /no).

A los últimos dos ítems se le asignó 1 punto y a los noes 2 puntos. De esta forma se pudieron sumar los datos de ambos ítems para generar una variable derivada sobre el grado de experiencia de los participantes con adultos mayores: 1 (experiencia parcial: o cuidando, o conviviendo), 2 (con experiencia), 3 (experiencia limitada: cuidando ancianos y conviviendo con ancianos), 4 (ninguna experiencia).

El cuestionario puede consultarse en el siguiente enlace: https://docs.google.com/forms/d/1tk_1e8XkaFB5dgKo0Jt2GOFfPMejy7HwaXTtB0rY OjQ/viewform

El cuestionario, diseñado como instrumento de recogida de datos útiles para el estudio, se configuró como un recurso no estandarizado, a diferencia de los test. No obstante, para valorar su potencial psicométrico, se calculó el coeficiente de consistencia interna de Cronbach y se realizó un análisis de componentes principales con carácter exploratorio.

La fiabilidad global de la escala de los 16 ítems fue relativamente baja, con un alpha de Cronbach de .54. Esta consistencia sólo mejoraba a .59 si se eliminaba el ítem número 14 (tabla "Tabla"). Por otro lado, se ha llevado un análisis de componentes principales con carácter exploratorio. Esta prueba es factible al tener suficiente varianza común entre las variables observadas, tal como ponen de manifiesto los indicadores de adecuación muestral Keiser-Meyer-Olkin (.624), la prueba de esfericidad de Barlett (Chi-cuadrado=293.00; g.l.=120; sig.=.000).

Se aplicó una rotación varimax, para buscar una máxima simplicidad en la estructura, puesto que este procedimiento maximiza la varianza de los coeficientes factoriales cuadrados en cada componente, y minimiza la posible correlación entre componentes. Se aplica también el criterio de seleccionar para autovalores iguales o mayor a 1, de forma que al menos el componente explique la varianza total de una variable. 
Tabla 1: Matriz de componentes rotados

\begin{tabular}{|c|c|c|c|c|c|c|}
\hline & & & Com & nente & & \\
\hline & 1 & 2 & 3 & 4 & 5 & 6 \\
\hline 5.- Las personas mayores suelen ser poco eficientes & 0.7 & & & & & \\
\hline 2.- Las personas mayores son productivas & 0.67 & & & & & \\
\hline 1.- Las personas mayores son independientes & 0.65 & & & & & \\
\hline 8.- Las personas mayores suelen ser activas & 0.52 & & 0.43 & & & \\
\hline 11.- Las personas mayores suelen ser poco hábiles & 0.5 & 0.4 & & & & \\
\hline $\begin{array}{l}\text { 13.- Las personas mayores suelen mostrarse } \\
\text { desconfiadas }\end{array}$ & & 0.68 & & & & \\
\hline 6.- Las personas mayores resisten poco físicamente & & 0.63 & & & & \\
\hline $\begin{array}{l}\text { 10.- Las personas mayores suelen ser sexualmente } \\
\text { activas }\end{array}$ & & 0.62 & 0.41 & & & \\
\hline $\begin{array}{l}\text { 7.- Las personas mayores } \\
\text { ideológicamente progresistas }\end{array}$ & & & 0.72 & & & \\
\hline 3.- las personas mayores suelen estar sanas & & & 0.71 & & & \\
\hline 15.- las personas mayores so poco sociables & & & & 0.76 & & \\
\hline $\begin{array}{l}\text { 12.- las personas mayores se integran poco } \\
\text { socialmente }\end{array}$ & & & & 0.76 & & \\
\hline $\begin{array}{l}\text { 4.- Las personas mayores suelen mostrarse } \\
\text { intolerantes }\end{array}$ & & & & & & \\
\hline 16.- Las personas mayores se encuentran valoradas & & & & & 0.85 & \\
\hline 9.- Las personas mayores suelen estar protegidas & & & & & 0.65 & 0.48 \\
\hline 14.- las personas mayores no son conflictivas & & & & & & 0.87 \\
\hline
\end{tabular}

En tabla 1 de componentes, se pueden observar los pesos para cada variable en cada componente, determinando así el significado que caracteriza a cada componente. Se han eliminado los pesos inferiores a 0.4 para facilitar su lectura. En este sentido, la magnitud expresa la correlación de cada variable con el componente. La varianza total explicada por el modelo fue de $60.59 \%$.

\section{Análisis}

Sobre los datos obtenidos se llevó a cabo un análisis descriptivo para medidas ordinales (frecuencia, mediana y moda, así como el rango intercuartílico). Igualmente, se calcularon los estadísticos "media" y "desviación típica" aunque no son adecuados para este tipo de medida. En cualquier caso, se incluyeron por ser habitual su utilización con carácter orientativo en las publicaciones de Ciencias de la Educación.

También se exploraron posibles diferencias en función del género y de la experiencia con adultos mayores sobre las puntuaciones obtenidas de cada bloque de ítems. Puesto que el objetivo era recoger información de las distintas afirmaciones (ítems9 de la escala, se prefirió trabajar con las respuestas a los ítems que con las puntuaciones de los componentes latentes. De esta forma, se pretendió ser coherente con el objetivo inicial, haciendo predominar la utilidad de la información recogida con 
el cuestionario, sobre las propiedades psicométricas del mismo. Para ello se utilizó el análisis de varianza unifactorial. El nivel de significación de referencia fue de alfa igual a .05. Todos los análisis se realizaron con el programa estadístico SPSS versión 22.

\section{Resultados}

\section{Experiencia con adultos mayores}

El 74,5\% de los participantes no tienen experiencia con mayores, tal como se ha medido en el cuestionario (variable derivada de la suma de puntuaciones del ítem "Viven contigo tus abuelos...” y “¿Has trabajado cuidando...?”). Por su parte, el 24.9\% presenta una experiencia parcial: $18.8 \%$ conviven con sus abuelos o algún anciano, y el $6.1 \%$ ha tenido experiencia como cuidador.

\section{Relaciones sociales}

En la tabla 2 se presentan los porcentajes de elección de cada opción junto con los estadísticos descriptivos básicos, así como el sentido del ítem (a favor o en contra del significado del bloque de ítems). A continuación, se comentan los resultados sumando las opciones 1 y 2 por un lado, junto con la 4 y 5 por otro, estableciendo así una comparativa entre los participantes a favor y en contra de cada ítem.

En el ítem 1 sobre independencia, los participantes se dividen en dos subgrupos parecidos. Los que están a favor de que los mayores son independientes (38.5\%) frente a los que piensan lo contrario (36.1\%). Con relación a la intolerancia (ítem 4) los participantes se vuelven a dividir en dos grupos, si bien, en este caso, hay una mayoría que se expresa en contra del ítem (45.5\%) frente a un 35.5\% a favor.

Tabla 2: Bloque de ítems sobre relaciones sociales (\% de respuestas)

\begin{tabular}{lcccccccccc}
\hline Ítem & 1 & 2 & 3 & 4 & 5 & s/o & Md & Media & Sx & Sentido \\
\hline $\begin{array}{l}\text { 1.- Las personas mayores son } \\
\text { independientes }\end{array}$ & 1.8 & 34.3 & 24.3 & 35.3 & 3 & & 2 & 3.04 & 0.95 & + \\
\hline $\begin{array}{l}\text { 4.- Las personas mayores suelen } \\
\text { mostrarse intolerantes }\end{array}$ & 5.9 & 39.6 & 18.3 & 33.7 & 1.8 & 0.6 & 2 & 2.86 & 1.02 & - \\
\hline $\begin{array}{l}\text { 9.- Las personas mayores suelen } \\
\text { estar protegidas }\end{array}$ & 1.8 & 32 & 11.2 & 42.6 & 7.7 & 4.7 & 2 & 3.24 & 1.06 & + \\
\hline $\begin{array}{l}\text { 12.- Las personas mayores se } \\
\text { integran poco socialmente }\end{array}$ & 32.5 & 55 & 4.7 & 4.1 & 1.8 & 1.2 & 1 & 1.86 & 0.83 & - \\
\hline $\begin{array}{l}\text { 14.- Las personas mayores no son } \\
\text { conflictivas }\end{array}$ & 4.1 & 24.9 & 18.9 & 36.7 & 13 & 2.4 & 2 & 3.30 & 1.12 & + \\
\hline $\begin{array}{l}\text { 15. - Las personas mayores son poco } \\
\text { sociables }\end{array}$ & 32.5 & 57.4 & 3.6 & 4.1 & 1.8 & 0.6 & 1 & 1.85 & 0.82 & - \\
\hline $\begin{array}{l}\text { 16.- Las personas mayores se } \\
\text { encuentran valoradas }\end{array}$ & 17.2 & 42.6 & 13.6 & 15.4 & 7.1 & 3.6 & 2 & 2.51 & 1.18 & + \\
\hline
\end{tabular}


Nota: 1 (totalmente en desacuerdo); 2 (de acuerdo); 3 (no estoy seguro); 4 (de acuerdo); 5 (totalmente de acuerdo); s/o (sin opinión); Sx (desviación típica); Md: Mediana; Sentido (+ a favor de las relaciones sociales, - en contra de las relaciones sociales).

Cuando los participantes se expresan con relación al nivel de protección (social) de las personas mayores, la mayoría está de acuerdo en afirmar que están protegidos (50.3\%). No obstante, destaca que el $33.8 \%$ piense lo contrario, así como un $4.7 \%$ que indica que no tiene una opinión formada al respecto.

Con relación al ítem 12, la amplia mayoría de los participantes se manifiestan en contra. Obsérvese que el ítem tiene un sentido contrario las relaciones sociales en los mayores (ver tabla 2).

Respecto a los tres últimos ítems, la mayoría de los encuestados se posiciona a favor del ítem 14 con el 49.7\%. Esta respuesta es coherente con la recogida en el ítem 15, donde una amplia mayoría del $89.9 \%$ está en contra, o totalmente en contra, de considerar a los mayores como poco sociables. Por último, los encuestados consideran mayoritariamente (59.9\%) que los mayores no se encuentran valorados (ítem 16).

\section{Actividad productiva}

La mayoría de los participantes en el estudio están de acuerdo en afirmar que las personas mayores son productivas (ver tabla 3). Esta respuesta es coherente con los datos recogidos en el ítem 5 (eficiencia) donde el 72.8\% están totalmente en desacuerdo o simplemente en desacuerdo, en considerar a los mayores como poco eficientes.

Tabla 3: Bloque de ítems sobre actividad productiva (\% de respuestas)

\begin{tabular}{llllllllllll}
\hline Ítem & 1 & 2 & 3 & 4 & 5 & s/o & Md & Media & Sx & $\begin{array}{c}\text { Sen- } \\
\text { tido }\end{array}$ \\
\hline $\begin{array}{l}\text { 2. - Las personas mayores son } \\
\text { productivas }\end{array}$ & 0.6 & 8.9 & 10.1 & 56.2 & 21.9 & 1.8 & 4 & 3.92 & 0.86 & + \\
$\begin{array}{l}\text { 5.- Las personas mayores suelen ser } \\
\text { poco eficientes }\end{array}$ & 15.4 & 57.4 & 14.2 & 10.7 & 1.2 & 1.2 & 2 & 2.24 & 0.88 & - \\
$\begin{array}{l}\text { 8.- Las personas mayores suelen ser } \\
\text { activas }\end{array}$ & 0.6 & 32.5 & 21.9 & 37.9 & 5.3 & 1.8 & 2 & 3.15 & 0.97 & + \\
$\begin{array}{l}\text { 11.- Las personas mayores se muestran } \\
\text { poco hábiles }\end{array}$ & 4.1 & 46.7 & 12.4 & 32 & 3.6 & & 2 & 2.84 & 1.04 & - \\
\hline
\end{tabular}

Nota: 1 (totalmente en desacuerdo); 2 (de acuerdo); 3 (no estoy seguro); 4 (de acuerdo); 5 (totalmente de acuerdo); s/o (sin opinión); Sx (desviación típica); Md: Mediana; Sentido (+ a favor de las relaciones sociales, - en contra de las relaciones sociales).

Sin embargo, como se puede observar en las respuestas dadas al ítem 8 (tabla 3) entre los encuestados no existe unanimidad a la hora de considerar a los mayores como personas activas. Así, el $33.1 \%$ se expresa contra esa idea frente a un $43.1 \%$ que se expresa de acuerdo o totalmente de acuerdo con el ítem. Igualmente, aunque menos acentuada, se ha registrado esta bipolaridad de opiniones con relación al ítem 11 (tabla $3)$. 


\section{Salud}

Con relación a la salud, la mayoría de participantes se expresan en contra de considerar que las personas mayores están sanas. Tan sólo un $20.7 \%$ está de acuerdo con el ítem 3 (tabla 4). En esta misma línea argumental, la mayoría de encuestados $(52.7 \%)$ considera que las personas mayores tienen poca resistencia física. Además, una mayoría del $49.1 \%$ considera que las personas mayores no son sexualmente activas (ítem 10). En este mismo ítem destaca que un 26\% eligen la opción 3 (no estoy seguro) junto con un $13 \%$ que afirma no tener opinión al respecto.

Tabla 4: Bloque de ítems sobre salud (\% de respuestas)

\begin{tabular}{|c|c|c|c|c|c|c|c|c|c|c|}
\hline Ítem & 1 & 2 & 3 & 4 & 5 & s/o & Md & Media & Sx & Sentido \\
\hline $\begin{array}{l}\text { 3.- Las personas mayores suelen estar } \\
\text { sanas }\end{array}$ & 3.6 & 53.3 & 22.5 & 19.5 & 1.2 & & 2 & 2.62 & 0.88 & + \\
\hline $\begin{array}{l}\text { 6.- Las personas mayores resisten } \\
\text { físicamente poco }\end{array}$ & 3 & 29.6 & 13 & 42 & 10.7 & & 2 & 3.28 & 1.10 & - \\
\hline $\begin{array}{l}\text { 10.- Las personas mayores suelen ser } \\
\text { sexualmente activas }\end{array}$ & & 41.4 & 26.6 & 10.1 & 0.6 & 13 & 2 & 2.47 & 0.84 & + \\
\hline
\end{tabular}

Nota: Leyenda: 1 (totalmente en desacuerdo); 2 (de acuerdo); 3 (no estoy seguro); 4 (de acuerdo); 5 (totalmente de acuerdo); s/o (sin opinión); Sx (desviación típica); Md: Mediana; Sentido (+ a favor de las relaciones sociales, - en contra de las relaciones sociales).

\section{Aspectos cognitivos}

Con relación a estos dos aspectos, las personas encuestadas se expresan mayoritariamente en contra de ver a las personas mayores como progresistas, pero también en verlas como desconfiadas (tabla 5).

Tabla 5: Bloque de ítems sobre aspectos cognitivos (\% de respuestas)

\begin{tabular}{llllllllllllll}
\hline Ítem & & 1 & 2 & 3 & 4 & 5 & s/o & Md & Media & Sx & Sentido \\
$\begin{array}{l}\text { 7.- Las personas mayores suelen } \\
\text { ideológicamente progresistas }\end{array}$ & ser & 5.3 & 40.2 & 26.6 & 17.8 & 3 & 6.5 & 2 & 2.71 & 0.95 & + \\
$\begin{array}{l}\text { 13.- Las personas mayores } \\
\text { mostrarse desconfiadas }\end{array}$ & suelen & 8.9 & 43.2 & 7.7 & 33.1 & 4.7 & 2.4 & 2 & 2.81 & 1.15 & - \\
\end{tabular}

Nota: 1 (totalmente en desacuerdo); 2 (de acuerdo); 3 (no estoy seguro); 4 (de acuerdo); 5 (totalmente de acuerdo); s/o (sin opinión); Sx (desviación típica); Md: Mediana; Sentido (+ a favor de las relaciones sociales, - en contra de las relaciones sociales). 


\section{Análisis descriptivo puntuaciones finales}

Las puntuaciones de los participantes se sumaron para cada uno de los bloques de ítems. Los resultados pueden observarse en la tabla 6. Destacan la presencia de ligeros sesgos hacia la derecha en las dimensiones RRSS (relaciones sociales) y Salud tal como se puede observar en la asimetría en la tabla 6. Por su parte, la dimensión de actividad productiva (Actividad) presenta un ligero sesgo hacia la izquierda.

Tabla 6: Bloque de ítems sobre aspectos cognitivos

\begin{tabular}{lcccccccc}
\hline Variable & $\mathrm{N}$ & Media & $\begin{array}{l}\text { Err.Est. } \\
\text { Media }\end{array}$ & $\begin{array}{l}\text { Desv } \\
\text { Std }\end{array}$ & Curtosis & Asimetría & $\begin{array}{l}\text { Mínimo de } \\
\text { la escala }\end{array}$ & $\begin{array}{l}\text { Máximo } \\
\text { de la escala }\end{array}$ \\
\hline RRSS & 147 & 5.50 & .24 & 2.89 & -.37 & .23 & -11 & 12 \\
\hline Actividad & 161 & 1.99 & .19 & 2.46 & -.48 & -.28 & -8 & 8 \\
\hline Salud & 145 & 1.74 & .17 & 2.05 & -.52 & .31 & -3 & 9 \\
\hline Cognición & 153 & -.11 & .12 & 1.46 & -.27 & .01 & -4 & 4 \\
\hline
\end{tabular}

Nota: casos válidos $=169$.

\section{Diferencias entre grupos}

Se aplicaron distintos análisis de varianza (ANOVA) para tratar de identificar diferencias estadísticamente significativas entre hombres y mujeres, así como diferencias en función del grado de experiencia con mayores. Los resultados no mostraron diferencias en función de la experiencia.

Con relación al sexo se registraron diferencias significativas con relación al bloque de Salud y Cognición (tabla 7). En ambos casos se comprobó la homocedasticidad de las varianzas.

Tabla 7: ANOVA de un factor en función de la experiencia con mayores

\begin{tabular}{lllllll}
\hline & & Suma de Cuadrados & df & Cuadrado medio & F & Sign. \\
\hline Salud & Entre Grupos & 26.08 & 1 & 26.08 & 6.44 & .012 \\
\hline & Intra Grupos & 579.48 & 143 & 4.05 & & \\
\hline Cognicion & Eotal & 605.56 & 144 & & & \\
\hline & Intre Grupos & 13.18 & 1 & 13.18 & .012 \\
\hline & Total & 323.11 & 151 & 2.05 & & \\
\hline
\end{tabular}

En el caso del bloque de Salud, los hombres presentan una media de 0.71 (d.t.= 2.35) frente a las mujeres, con una media superior de 1.92 (d.t.=1.95). Por su parte, con relación a la dimensión de Cognición, la media de los hombres es de -0.94 (d.t.=1.78) mientras que en las mujeres es de -0.01 (d.t. =1.39). 
Estas diferencias significativas se pueden apreciar también en los histogramas de cada segmento (figura 1).

Figura 1: histogramas de las dimensiones Salud y Cognición en función del sexo

Salud hombres:

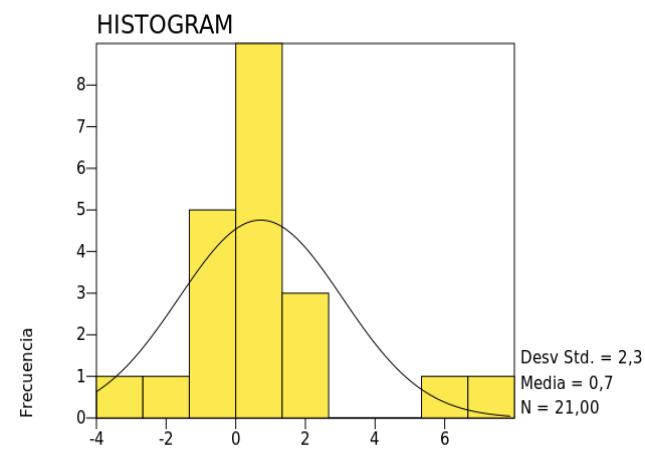

salud

Salud mujeres:

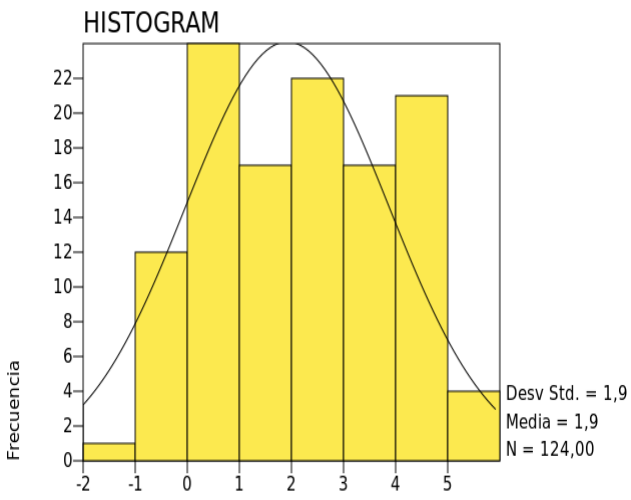

salud
Cognición hombres:

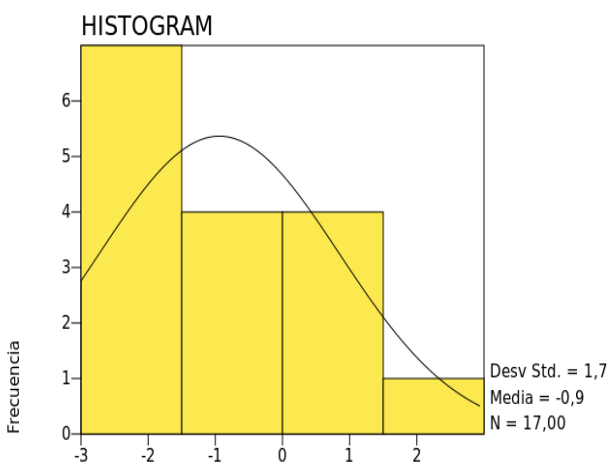

cognicion

Cognición mujeres:

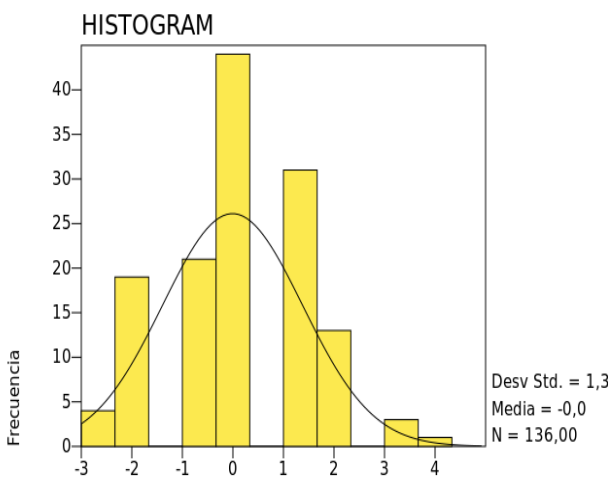

cognicion

\section{Perfil del mayor según los jóvenes}

Tras obtener los resultados anteriores se realizó un análisis descriptivo independiente en hombres y mujeres de la muestra. De esta forma se pudo obtener un perfil descriptivo de la representación mental de los adultos mayores en función del sexo de los jóvenes que participaron en el estudio (tabla 8). 
Tabla 8: Perfil del adulto mayor según los jóvenes encuestados

\begin{tabular}{llllllll}
\hline & \multicolumn{2}{l}{ Según hombres } & & Según mujeres & & $\begin{array}{c}\text { Mínimo } \\
\text { de la } \\
\text { escala }\end{array}$ \\
Variable & Mínimo & Media & Máximo & Mínimo & Media & Máximo & \\
RRSS & -1.00 & 5.50 & 11.00 & .00 & 5.50 & 12.00 & -11 \\
Actividad & -3.00 & 1.05 & 7.00 & -4.00 & 2.13 & 8.00 & -8 \\
\hline Salud & -3.00 & .71 & 7.00 & -2.00 & 1.92 & 6.00 & -3 \\
\hline Cognición & -3.00 & -.94 & 3.00 & -3.00 & -.01 & 4.00 & -4 \\
\hline
\end{tabular}

Para facilitar la utilización de estos resultados con el fin de comparar las variables entre sí, se tipificaron los datos de las distintas dimensiones y se calcularon distintos percentiles (tabla 9).

Tabla 9: Percentiles de cada variable por sexo en puntuaciones típicas

\begin{tabular}{lllllllll} 
& Percentiles: & 5 & 10 & 25 & 50 & 75 & 90 & 95 \\
Relaciones sociales & Hombre & -1.61 & -1.46 & -0.47 & -0.16 & .47 & 1.71 & 1.71 \\
\hline & Mujer & -1.58 & -1.23 & -0.88 & -0.18 & .53 & 1.58 & 1.93 \\
\hline Actividad & Hombre & -1.22 & -1.30 & -1.15 & -0.02 & .74 & 2.02 & 2.24 \\
\hline \multirow{2}{*}{ Salud } & Mujer & -1.71 & -1.30 & -0.47 & -0.05 & .78 & 1.19 & 1.61 \\
\hline \multirow{2}{*}{ Cognición } & Hombre & -1.27 & -1.33 & -0.73 & .12 & .55 & 2.42 & 2.68 \\
\hline & Mujer & -1.49 & -0.98 & -0.98 & .04 & 1.07 & 1.27 & 2.09 \\
\hline & Hombre & -0.92 & -1.15 & -0.59 & -0.03 & .53 & 1.87 & 2.21 \\
\hline & Mujer & -1.44 & -1.44 & -0.72 & .01 & .73 & 1.45 & 1.45 \\
\hline
\end{tabular}

\section{Discusión}

En primer lugar se quisiera señalar el relativo bajo porcentaje de participantes que tenía experiencia con adultos mayores. Esta experiencia, según la literatura consultada, podría estar mediando en la percepción que los jóvenes tienen sobre este segmento de población y sobre el propio proceso de envejecimiento. Una mayor experiencia o contacto con los adultos mayores parecen fomentar una visión más positiva en los jóvenes (Van Dussena \& Weaverb, 2009).

Con relación a los distintos ítems de la escala, los participantes del estudio conciben al adulto mayor como alguien social. En la dimensión de las relaciones sociales, no parece haber unanimidad con relación a la independencia, encontrándose dos grupos casi iguales. Estos dos grupos no parecen determinarse en función del sexo o la experiencia. Por tanto, sería interesante realizar nuevos estudios que traten de averiguar en qué se basa la percepción de dependencia/independencia en esta muestra. 
Respecto a la dimensión de actividad, la mayoría de los encuestados están de acuerdo en considerar a los adultos mayores como productivos. Estos resultados son similares a los encontrados en muestras de estudiantes chilenos (Sahuenza, 2014). En este mismo sentido, los encuestados conciben a los adultos mayores como eficientes. Estas perspectivas pueden estar ligadas a un enfoque economicista de la realidad del adulto mayor. La prolongación de la vida laboral, así como la comercialización de nuevos servicios y productos específicos para los adultos mayores no ha pasado desapercibido para los países desarrollados. En este sentido, la Unión Europea está publicando desde hace algún tiempo, distintos informes bajo la expresión de Silver Economy (https://ec.europa.eu/digital-agenda/en/news/growing-silver-economybackground-paper). Puesto que la mayoría de participantes piensa que los mayores son productivos, un próximo estudio debería identificar para qué o en qué áreas son productivos.

A pesar de esta visión generalizada, en los resultados de la escala destaca que los encuestados no son unánimes sobre si los adultos mayores son activos o no. En este sentido, deberían hacerse más estudios para analizar si esta opinión está mediada por alguna variable contextual.

Con relación a la salud, la idea generalizada es que las personas mayores no están sanas y que tienen una resitencia física limitada. También destaca la idea de que no existe vida sexual en esta etapa de la vida. Sin embargo, aunque son resultados coherentes con otros estudios, los porcentajes de opinión en este sentido son más bajos que en dichos estudios que rondan el setenta por ciento (Arnold-Cathalifaud, Thumala, Urquiza \& Ojeda, 2007; Sahuenza, 2014). No obstante, dicha diferencia de porcentaje es menor si se tiene encuenta también los encuestados del presente estudio que eligieron la opción intermedia. También destaca que un relativo alto número de encuestados manifestó que no tenía una opinión formada sobre esta cuestión.

Por último, con relación a la dimensión cognitiva, la idea generalizada es que el adulto mayor es poco progresista y desconfiado. No obstante, con relación a este último aspecto, la opinión está relativamente dividida.

Que las personas encuestadas se expresen mayoritariamente en contra de ver a las personas mayores como progresistas, pero también en verlas como desconfiadas, también puede sugerir que ha existido una falta de comprensión de los ítems por parte de los participantes. En este sentido, será necesario una revisión de los ítems que operativizan esta dimensión.

Por otro lado, se ha llevado a cabo un contraste de hipótesis en función de la experiencia y del sexo. Con relación al primer factor no se han encontrado diferencias. Este resultado pone en cuestión estudios previos como los llevados a cabo por Van Dussena y Weaverb (2009). Este es otro de los aspectos que deberían indagarse con mayor profundidad en futuros estudios.

Respecto al sexo de los participantes, sólo se han encontrado diferencia en la 
dimensión de salud y cognición. Los gráficos muestran que los hombres tienden a tener una opinión más homogénea, aunque algo más negativa, que las mujeres.

Finalmente, haciendo una valoración general de los resultados, se puede afirmar que la representación general del adulto mayor en los estudiantes encuestados es relativamente positiva, lo que no coincide en general con la obtenida en otros estudios de la literatura consultada. Así, se caracterizaría por una visión relativamente positiva en las relaciones sociales y la actividad productiva, tanto para los hombres como para las mujeres consultadas. Con relación a los aspectos de ideología y confianza la representación está en un punto medio. Sin embargo, los adultos mayores se conciben con una salud deficiente, si bien esto es más negativo para los hombres encuestados que para las mujeres.

Esto resultados tienen implicaciones tanto para los jóvenes, en la medida que serán los futuros adultos mayores, como para los adultos mayores actuales. Dichas implicaciones, especialmente en el ámbito educativo, se comentan en el siguiente epígrafe.

\section{Conclusiones}

Con relación al objetivo de la investigación, se ha conseguido un perfil de la representación social que el adulto mayor tiene para los estudiantes de Educación de la Universidad de Málaga. De forma resumida, el adulto mayor para los encuestados se caracteriza, en términos generales, por:

- Ser sociable.

- Ser productivamente activo.

- Tener problemas de salud.

- Cierta tendencia a evitar ideas progresistas y algo desconfiado.

Aunque el perfil es una generalización de los resultados, cabe destacar varias cuestiones al respecto. La primera que no es tan negativo como estudios consultados con anterioridad, tal como se ha debatido en la discusión. La segunda, que pone de manifiesto que la dimensión percibida como más negativa es la relacionada con la salud.

Si esta visión sobre la salud permanece en los jóvenes estudiantes, es razonable suponer que puede afectarles a medida que envejecen, generando un rechazo ante esta etapa de la vida. Esto puede afectar a la visión que tengan de sí mismos. Es decir, las imágenes negativas pueden generar un sesgo en la autopercepción, provocando un efecto de profecía autocumplida. Todo ello está vinculado a la autoestima y la depresión. Sin duda, esto puede estar ocurriendo con buena parte de los adultos mayores actuales. 
Por otro lado, las representaciones sociales determinan la forma de interactuar entre los miembros de una sociedad. En este sentido, una perspectiva negativa del envejecimiento puede generar un patrón de comportamiento paternalista hacia el adulto mayor.

Por el contrario, una visión más realista del envejecimiento lleva a la sociedad a un planteamiento más positivo. En gran medida, estos planteamientos suponen una perspectiva más coherente con el envejecimiento activo.

En este sentido, la identificación de sesgos en la representación del adulto mayor en otros segmentos de la población permitirá intervenir para su corrección. De esta forma, se evitará repetir comportamientos disfuncionales en las próximas generaciones de adultos mayores, y con ello, algunos de los problemas sociales de sobredependencia.

Esta forma de entender el envejecimiento y el papel del adulto mayor, lleva implícita la necesidad de satisfacer una serie de necesidades. Entre estas necesidades se encuentran la del aprendizaje continuo a lo largo de la vida. Dentro de este marco es donde también adquiere sentido la identificación de sesgos en la representación social de los estudiantes de Educación con relación a los adultos mayores.

Los estudiantes de Educación deberán estar preparados para atender estas necesidades formativas de los adultos mayores. Por ello necesitan programas formativos que los preparen para afrontar dichas tareas.

Según se desprende de los resultados del presente estudio, estos programas formativos deben afrontar la diferencia de ideas preconcebidas en función del sexo, con relación a los adultos mayores.

Por otro lado, y aunque el presente estudio no ha encontrado diferencias en función de la experiencia con los mayores, deben tenerse algunos estudios internacionales cuyos resultados sí apuntan a la importancia de este factor. En este sentido, los programas formativos deberían diseñarse tratando de fomentar la interacción intergeneracional.

Por último, es necesario señalar que, si bien los resultados de este estudio han sido muy útiles para la población diana, deben tomarse con precaución si se pretenden utilizar en otra población. En este sentido, estudios posteriores deberían realizarse con muestras más amplias y construidas con sistemas que garanticen su representatividad sobre las poblaciones de interés.

Otra limitación del estudio los constituye la propia escala utilizada. Si bien ha reflejado bastante bien las dimensiones básicas de interés, es necesario señalar que el número de ítems para algunas de ellas es bastante bajo. En este sentido, estudios posteriores deberían enriquecer el instrumento con un mayor número de ítems, equilibrando el mismo en todas las dimensiones. 


\section{Referencias}

Abades, M., \& Rayón, E. (2012). El envejecimiento en España: ¿un reto o problema social? Gerokomos, 23(4), 151-155.

Abellán, A., Vilches, R. \&Pujol, R. (2014). Un perfil de las personas mayores en España, 2014. Indicadores estadísticos básicos. Informes, envejecimiento en red, 6, 1-24. Recuperado de http://envejecimiento.csic.es/documentos/documentos/enredindicadoresbasicos14.pdf

Adelantado, F., Segura, C., De Andrés, J., Feliú, T., \& Martínez, P. (2004). Los mayores de 85 años en Sabadell. Revista Multidisciplinar de Gerontología, 14(5), 271-279.

Aristizábal-Vallejo, N. (2005). Imagen social de los mayores en estudiantes jóvenes universitarios. Pensamiento Psicológico, 5, 27-32.

Arnold-Cathalifua, M., Thumala, D., Urquiza, A. \& Ojeda, A. (2007). La vejez desde la mirada de los jóvenes chilenos: estudio exploratorio. Última Década, 27, 7591.

Baka, A., \& Figgou, L. (2012). 'Neither agree, nor disagree': a critical analysis of the middle answer category in Voting Advice Applications. International Journal Governance, 5(3/4), 244-263.

Birkenmaier, J., Damron-Rodríguez, J. \& Rowan, N. (2009). Social work knowledge of facts on aging: Influence of fiel dan classroom education. Educational Gerotology, 35(9), 784-800.

Cherry, K. \& Palmore, E. (2008). Relating to older people evaluation (ROPE): A measure of self-reported ageism. Educational Gerontology, 34(10), 849-861.

Díaz, A, Fernández, M, Gázquez, J, González, L, Pérez-Fuentes, M \& Ruíz, I. (2009). Oldage stereotypes related to gerontology education: An intergenerational study. European Journal of Education and Psychology, 2(3), 263-273.

Fealy, G, Lyons, I, McNamara, M \& Treacy, M. (2012). Constructing ageing and age identities: a case study of newspaper discourses. Ageing \& Society, 32(1), 85102.

Futurage (2011). A Road Map for European Ageing Research. Sheffield: Univeridad de Sheffield. Recuperado de http://futurage.group.shef.ac.uk/

Gómez Carroza, T. (2003). Heteroestereotipos y autoestereotipos asociados a la vejez en Extremadura. Tesis Doctoral. Universidad de Extremadura, Facultad de Formación del Profesorado, Departamento de Psicología y Sociología de la Educación, Cáceres. 
INE (2014) Proyección de la Población de España 2014-2064 [ebook]. Madrid: Instituto Nacional de Estadística, pp.1-15. Recuperado de http://www.ine.es/prensa/np870.pdf

Lachman, M \& Yun, R. (2006). Perceptions of aging in two cultures: Korean and american views on old age. Journal of Cross-Cultural Gerontology, 21(1), 5570.

Lentini, D, Ruiz, M \& Scipioni, A. (2008). Vejez e imaginario social. Revista Electrónica de Psicología Social, 6(16), 1-12.

Likert, R. (1932). A technique for the measurement of attitude. Archives of Psychology, 140, 5-55.

Matas, A., Leiva, J., \& Franco, P.D. (2016). Análisis del uso de las Nuevas Tecnologías en población española de 45-54 años: previsión de necesidades formativas para un envejecimiento activo. Pixel-Bit, 48, 225-240.

Pérez Grande, M.D., García del Dujo, A. \& Martín García, A.V. (1999). Prejuicios, estereotipos y otras profecías autocumplidorasun reto para la educación intercultural. Pedagogía social: revista interuniversitaria, 3, 125-146.

Sahuenza, J. (2014). Imágenes sobre la vejez en jóvenes estudiantes de carreras sin formación gerontológica. Neurama, revista electrónica de psicogerontología $1(1), 6-14$.

Tan, P., Zhang, N., \& Fan. L. (2004). Students' attitude toward the elderly in the people's republic of China. Educational Gerontology, 30(4), 305-314.

Van Dussen, D \& Weaver, R. (2009). Undergraduate students' perceptions and behaviors related to the aged and to aging processes. Educational Gerontology, $35(4), 342-357$.

Zhou, L. (2007). What college students know about older adults: A cross-cultural qualitative study. Educational Gerontology, 33(10), 811-831.

Fecha de recepción:18/01/2016

Fecha de revisión: 12/05/2016

Fecha de aceptación: 13/10/2016 
Matas, A., Leiva, J.J., Franco, P.D. y Isequilla, E. 